\title{
PD-1 Knockout Aggravates Motor Dysfunction In The MPTP Model of Parkinson's Disease By Inducing Microglial Activation And Neuroinflammation In Mice
}

\author{
Ying-Ying Cheng \\ Fourth Military Medical University: Air Force Medical University \\ Bei-Yu Chen \\ Fourth Military Medical University: Air Force Medical University \\ Gan-Lan Bian \\ Fourth Military Medical University: Air Force Medical University \\ Yin-Xiu Ding \\ Ningxia Medical College: Ningxia Medical University \\ Liang-Wei CHEN ( $\square$ Iwchen@fmmu.edu.cn ) \\ Fourth Military Medical University
}

\section{Research}

Keywords: PD-1 signaling pathway, Microglial cell, Neuroinflammation, Parkinson's disease

Posted Date: July 27th, 2021

DOI: https://doi.org/10.21203/rs.3.rs-724934/v1

License: (c) (i) This work is licensed under a Creative Commons Attribution 4.0 International License.

Read Full License 


\section{Abstract}

Background: Abundant microglial reaction and neuroinflammation are typical pathogenetic hallmark of brains in Parkinson's disease (PD) patients, but regulation mechanisms are poorly understood. In this study, the promoting effects of PD-1-difficiency on microglial activation, neuroinflammation and motor dysfunction were identified using PD animal model.

Methods: Using C57 wild-type (WT), PD-1 knockout (KO) and MPTP model, we designed WT-control, KOcontrol, WT-MPTP and KO-MPTP groups. Motor dysfunction of animal, distribution of PD-1-positive cells, dopaminergic neuronal survival, glial cell activation and generation of inflammatory cytokines in midbrains were observed by behavior detection, immunohistochemistry and western blot methods.

Results: Microglial cells showing PD-1/lba1 double-positivity were numerously distributed in the substantia nigra of control whereas they decreased in MPTP model. Compared with WT-MPTP, KO-MPTP mice exacerbated in their motor dysfunction, decreased level of TH expression and decreased TH-positive neuronal protrusions. Microglial cell activation and expression of proinflammatory cytokine iNOS, TNF-a, IL-1 $\beta$ and IL- 6 significantly increased, and levels and phosphorylation of AKT and ERK1/2 were also elevated in KO-MPTP mice.

Conclusions: PD-1 knockout could aggravate motor dysfunction of MPTP mouse model by promoting microglial activation and neuroinflammation in midbrains, suggesting that PD-1 signaling abnormality might be involved in PD pathogenesis or progression.

\section{Background}

Parkinson's disease (PD) is a common and severe degenerative disease in the central nervous system (CNS) with high incidence which is secondly to Alzheimer's disease (AD). The typical pathological feature of $\mathrm{PD}$ is degenerative death of dopaminergic neurons in the nigra-striatal system that results in severe dopamine-deficiency in the brains and motor dysfunction (1-4). Accumulating studies have shown that neuroinflammation dominated by activated glial cells, i.e. reactive microglial cells and activated astrocytes in the CNS, is involved in inducement of dopaminergic neuronal degeneration in PD progression (5-8). Under physiological condition, obviously, microglial cells and astrocytes work in protection of neurons and assist synaptic transmission, remove dying cell fragments or foreign harmful molecules, secrete neurotrophic factors and stabilize microenvironment of central neurons (9-11). Reactive microglial cells and astrocytes join neuroinflammation and neuronal damage, and also constitute pathological characteristics of $A D, P D$, multiple sclerosis, traumatic brain injury and other infectious neuropathy states $(4-8,12-14)$. However, detailed significance of inflammatory response and related key regulation factors in modulating glial activation or functional transition from congenital immunity to neuroinflammation state are still unclear $(14,15)$.

Programmed death receptor-1 (PD-1) is a key checkpoint molecule of immune function, which is a membrane protein expressed in a variety of immune cells such as lymphatic T cells, B cells, monocytes, 
natural killer cells, and plays an important roles in regulating antigen response or limiting host adaptive immune response by threshold of $T$ cells and $B$ cells, and thereafter joining in prevention of autoinflammation response or diseases (16-18). PD-1 ligands include PD-L1 and PD-L2 bind PD-1 receptor, initiate PD-1 signaling and followed cellular effects. In the tumor microenvironment and inflammatory state, expression of PD-1 in T-cell increases abnormally. PD-L1 then combines PD-1 and initiates a series of inhibition signaling, resulting in decline of T-cell function, causing immune tolerance of tumor cells and evading T-cell immune surveillance $(19,20)$. Therefore, PD-1/PD-L1 signaling pathway is critically in immune function regulation and immune tolerance, and provides a therapeutic way for immune modulatory therapy or intervention of various diseases (21-24).

It is well known that microglial cells belong to the monocyte-macrophage system based on their source of occurrence and characteristics, showing antigen presentation function and secretion of a variety of active factors. PD-L1 is identified in the glial cells and expressional change is observed under CNS inflammatory state $(24,25)$. However, it is still lack of studies on PD-1/PD-L1 working in the neurodegenerative diseases, especially relation to microglial activation and neuroinflammatory response. It is interesting to note that existing studies on PD-1 role in the experimental autoimmune encephalomyelitis and spinal cord injury, and it reveals that PD-1-deficiency results in an increased neuropathological inflammation (26-29). Based on literature evidences, we speculate that PD-1 signaling pathway may be also an potential regulating factor in the activated glial cells and inflammation and neuronal damage during pathogenesis of PD. Therefore, PD-1 knockout and MPTP mouse model were applied in this study, immunohistochemistry, western blot and motor behavior detection were used to observe effects of PD-1deficiency on glial cell activation, neuroinflammatory reaction and motor dysfunction of animals, for purpose to determine possible mechanistic involvement of PD-1 signaling pathway in PD pathogenesis or progression, which may thereafter provide a new basis for exploring immunotherapy potentially for PD.

\section{Materials And Methods}

\section{Experimental Animals and Reagents}

The C57BL/ 6 mice (wild-type) used in study were provided by the Experimental Animal Center of the Fourth Military Medical University, and PD-1 knockout mice were introduced as preservation materials (C57BL/6 PD1-KO, originally developed by Kyoto University, 1998) to Neurobiology Department of Fourth Military Medical University. Animal experiments were conducted in accordance with the National Institute of Health guide for the care and use of Laboratory animals (NIH Publications, eighth edition revised in 2012) and under the approval of Animal Experiment Administration Committee of the Fourth Military Medical University, and all efforts were performed to minimize animal number and their suffering in this study.

Experimental reagents: 1-methyl-4-phenyl-1,2,3,6-tetrahydropyridine (MPTP, Sigma) for animal model preparation, $4 \%$ formaldehyde for tissue fixation, OCT encapsulation agent, phosphate-buffered saline (PBS), $0.1 \%$ Triton-X-100, RIPA lysate, protease inhibitors, phosphatase inhibitors, BCA protein 
quantitative kits (Thermo), electrophoresis fluids, transmembrane fluids, TBST fluids, chemical luminescent developer fluids (ZETA) and DAPI nuclear counter staining fluids (Sigma).

Antibody and working dilutions: Primary and secondary antibodies were purchased from Company of Sigma, Cell Signaling Technology (CST), DAKO, Wako and Invitrogen. Primary antibodies included rabbit anti-inducible nitric oxide synthase (iNOS), tumor necrosis factor-a (TNF-a), interleukin-1 (IL-6), arginine-1 (ARG-1), tissue growth factor- $\beta$ (TGF- $\beta$ ), IL-4, IL-10, AKT, p-AKT, ERK1/2, P-REK1/2 (CST), rabbit anti-lba1 (Wako), $\beta$-actin (Sigma), rat anti-glial fibrillary acid protein (GFAP, DAKO), tyrosine hydroxylase (TH, Sigma), IL-1 $\beta$, PD-1, PD-L1 (CST) (1:1000 to 1:3000 dilutions, respectively). Second antibodies included Alexa Fluo594-labeled sheep anti-rabbit IgG, Alexa Fluo594-labeled sheep anti-rat IgG, Alexa Fluo488labeled sheep anti-rabbit IgG, Alexa Fluo488-labeled sheep anti-rat IgG, HRP-labeled sheep anti-rabbit IgG and HRP-labeled sheep anti-rat IgG (Invitrogen) (1:500 to 1:1000 dilutions, respectively).

\section{Preparation of MPTP animal model}

The C57BL/ 6 mice, Wild-Type and PD-1 knockout weighing $20 \pm 2 \mathrm{~g}$, male and female, were used. The animal were arranged in WT control (WT-CON), WT MPTP (WT-MPTP), PD-1 knockout control (KO-CON), and PD-1 knockout MPTP (KO-MPTP) group with 5-10 mice each $(n=5-10)$. In MPTP group (WT-MPTP and KO-MPTP group), MPTP $30 \mathrm{mg} / \mathrm{kg} /$ day was intraperitoneally injected for consecutive 5 days. In control group (WT-CON and KO-CON) same amount of $0.9 \%$ saline was given by an intraperitoneal injection. After motor behavior detection of animals, the perfusion-fixation and frozen sections of midbrains were performed for immunohistochemical staining or double-labeling, or sample extraction of fresh midbrain tissues were carried out for western blot respectively.

\section{Animal motor behavior detection}

The MPTP animal model were prepared at time-points of the 5th, 10th and 15th day, groups of WT-CON, KO-CON, WT-MPTP, KO-MPTP mice were tested for motor behavior. Using the boom experiment method, the movement time (Seconds) and the number of falls (per $5 \mathrm{~min}$ ) were observed and counted, and the movement dysfunction and changes in different experimental groups of mice were analyzed and shown.

\section{Immunohistochemistry}

Animals in injectable anaesthetic state, saline and fallowed $4 \%$ formaldehyde solution was perfused for brain fixation, the midbrains was put into $20 \%$ sucrose PB solution, soaked overnight to tissue subsidence, the midbrain samples containing nigral region were token for continuous frozen sections with 15-20 micron thickness. Sections were selected to be rinsed 3 times in $0.01 \mathrm{M}$ PBS buffer and then incubated in $0.1 \%$ Triton X-100-3\% donkey serum for $30 \mathrm{~min}$. Sections were incubated in primary antibody solution respectively for 24 hours at $4{ }^{\circ} \mathrm{C}$ and $1 \mathrm{~h}$ at room temperature. After rinsing 3 times in $0.01 \mathrm{M} \mathrm{PBS}$, sections were incubated in secondary fluorescence-labeled antibody solution for 4 hours at room temperature. Finally Midbrain sections were processed for DAPI nuclear counter-stain and observed under a laser scanning confocal microscope (LSCM, Olympus, FX-1000), and interested images were captured for demonstration representatively. 


\section{Western blot}

Western blot was performed as in our previous studies (Cheng et al., 2020). Fresh lysates from midbrain samples were made in lysis buffer (Beyotime Biotechnology). The protein concentrations were measured by $B C A$, and $10 \mu \mathrm{l}(2 \mu \mathrm{g} / \mu \mathrm{l})$ of sample solution was loaded for each panel. After separation in sodium dodecyl sulfate polyacrylamide gel electrophoresis (SDS-PAGE) gels (10\%), protein samples were transferred onto PVDF membranes (Millipore). Membranes were then blocked with $5 \%$ nonfat milk/TBST $\left(0.1 \%\right.$ Tween-20, TBS) for 1 hour and incubated with primary antibody respectively at $4^{\circ} \mathrm{C}$ overnight. The $\beta$-actin level was used as an internal control for immunoblot. After incubation with HRP-conjugated secondary antibody at room temperature for $1 \mathrm{~h}$, immunoblots were detected using an advanced ECL detection kit according to manufacturer's instruction. For re-probing, the transfer membrane was stripped by complete washing with stripping buffer (glycine-SDS buffer, $\mathrm{pH} 2.0$ ) for $15 \mathrm{~min}$, followed by TBST (pH 7.4) three times, and then processed for a standard probing process. Immunoblot images were acquired and analyzed using Bio-Rad's system. Quantification was performed and presented as a density ratio to internal control.

\section{Statistical Analysis}

Quantitative data on immuno-positive cell counts and immunoblots were shown by Mean \pm Standard Error (mean \pm SEM), and the differences between the groups were compared by Student $T$-test (between two groups), and One-way ANOVA and Dunnett post-test (between four groups) using GraphPad Prism (5.0) software. When the $P$-value of comparison between groups was less than 0.05 and considered to have statistical significance.

\section{Results}

\section{Distribution of microglial cells showing PD-1 immunoreactivity in the substantia nigra}

First, double immunofluorescence for PD-1/lba1, PD-1/GFAP and PD-1/NeuN was carried out to observe localization of PD-1-immunoreactivity in the microglial cells, astrocytes and neurons in midbrains under a laser scanning confocal microscope. PD-1/lba1 double-labeled microglial cells were observed and numerously distributed in the substantia nigra of mice, and PD-1-immunoreactive products were clearly and strongly localized in cell membrane of the microglial cells. On the other hand, PD-1/GFAP doublelabeled astrocytes, PD-1/NeuN double-labeled neuronal cells were not observed in the substantia nigra regions (Fig. 1). In addition, double immunofluorescence for PD-L1/Iba1, PD-L1/GFAP, PD-L1/NeuN was also performed to observe if any PD-L1, PD-1 ligand, was localized in the microglial cells, astrocytes and neurons, and it indicated that PD-L1 was also mainly located in the microglial cells rather than astrocytes and neurons (data not shown here). 


\section{Decrease of microglial cells with PD-1/lba1 double-labeling in the substantia nigra of MPTP model}

Then, immunohistochemstry was again applied to observe if any changes of PD-1/lba1 double-labeled microglial cells distributed in the substantia nigra occurred in animal model of Parkinson's disease. A sub-acute mouse model of Parkinson's disease i.e. MPTP $30 \mathrm{mg} / \mathrm{kg}$ for continuous 5 days was performed and followed by nigral sections and immunostaining. Both Iba1-immunopositive microglial cells and GFAP-positive astrocytes in MPTP model showed active morphology with an increase in the numbers, consistent with our previous observations. Compared with control group, the number of microglial cells with PD-1/lbal double-labeling decreased significantly in MPTP group, and immunostaining intensity of PD-1 also decreased obviously. Comparison of corresponding ngral staining regions in control group and MPTP group showed differences in their morphology and quantity of PD1/Ibal double-labeled cells (Fig. 2). Cell count on PD-1/lba1 double-labeled cells in the unit area of onesided nigral sections indicated that $280 \pm 32$ in control group and $189 \pm 26$ in MPTP group with significant statistical differences (Student T-test, $P<0.01$ ).

\section{Aggravation effect of PD-1 knockout on motor dysfunction and reduction of TH-positive protrusions in MPTP model}

After that, PD-1-knockout mice was introduced to assess effect of PD-1 on the motor function and survival of dopamine neurons in MPTP model by comparing animal movement functions (motion time and falling times) and immunohistochemical visualization of TH nigral neurons at time-points of the d5, d10, d15 in WT-CON, WT-MPTP, KO-CON, KO-MPTP group. Compared with WT-MPTP, movement dysfunction in KO-MPTP group was more severe, the performance was more slow, the movement time was obviously shortened, the falling times increased, or animals was unwilling to move or even stay in place. The WT-CON mice also showed a decrease in motion time compared to KO-CON group, but there was no significant change in the falling times (Fig. 3). Immunohistochemistry and Western blot were further carried out to show TH-positive neurons, neuronal protrusions and TH expression levels. The number of TH-positive neurons in WT-MPTP group decreased significantly compared with WT control, which was same as the previous observations. Compared with WT-MPTP group, there was no significant difference in number of TH-positive neurons, but KO-MPTP showed a decreased trend of TH-positive neuronal cell protrusions or processes than WT-MPTP group $(P<0.05)$ (Fig. 4A, B). Immunoblot confirmed that KO-MPTP mice showed a significant decrease in TH expression level compared with WT-MPTP group $(P<0.01)$ (Fig. 4C).

\section{Promotion effect of PD-1 knock-out on microglial activation and proinflammatory cytokine expression in MPTP model}

Furthermore, effects of PD-1 knockout state on glial cell activation, pre-inflammatory factor (M1 marker molecule) and anti-inflammatory factor (M2 marker molecule) expression levels were analyzed to 
evaluate PD-1 participation in neuroinflammatory response. The KO-MPTP mice had a significant increase in Iba1 expression compared to WT-MPTP group $(P<0.01)$, while expression of PD-1, PD-L1 and GFAP did not show significant changes $(P>0.05)$ (Fig. 5). Expressions of proinflammatory cytokine iNOS, TNF- $\alpha$, IL-1 $\beta$ and IL- 6 showed up-regulated trend in both KO-CON and KO-MPTP group. The KO-MPTP mice increased expression of iNOS, TNF-a, IL-1 $\beta$ and IL- 6 significantly compared to the WT-MPTP group. The KO-CON group also showed upward changes of iNOS, IL-1beta, and IL6 except of TNF-a compared to WT-CON group (Fig. 6). Double immunostaining showed positive localization of iNOS, TNF-a, IL-1 $\beta$ and IL6 in the microglial cells (data not shown). In addition, anti-inflammatory cytokine ARG-1, TGF- $\beta$, IL-4, IL-10 expression change trend was not fully consistent. Compared with WT-MPTP group, ARG-1 showed a significant downward change, TGF- $\beta$ and IL-4 no significant change, while IL-10 showed a clear upward trend in expression in KO-MPTP group (Fig. 7). These data indicated that PD-1 knockout increased abnormal glial activation and proinflammatory cytokine production, and might thereafter induce or aggravate neuroinflammatory response, dopaminergic neuronal damage and motor dysfunction in MPTP animal model.

\section{Motivation influence of PD-1 knockout on AKT and ERK1/2 signaling in MPTP model}

Finally, Western blot was applied to analyze possible cell signaling mechanism of PD-1 knockout affecting glial activation and inflammatory response by examining AKT, p-AKT, ERK1/2 and p-ERK1/2 expression in the substantia nigra of MPTP animal model. Compared with WT-MPTP group, p-AKT, tAKT, p-ERK1/2 and tERK1/2 showed an increase in the expression levels with statistical significant in KOMPTP group (Fig. 8). Compared with WT-MPTP, at the same time, the ratio of p-AKT to tAKT, or p-ERK1/2 to tERK1/2 also increased significantly in KO-MPTP group. Data showed that phosphorylation or activation level of AKT and ERK1/2 increased to a certain extent in KO-MPTP group, indicating that PD-1 knockout could possibly promote activation of AKT and ERK1/2 signaling pathways in MPTP model of PD. However, whether AKT, p-AKT, ERK1/2, p-ERK1/2 signal molecules were exactly or mainly localized in these activated microglial cells or astrocytes was subject to further experimental confirmation.

\section{Discussion}

In this study, PD-1 knockout mice and MPTP model of Parkinson's disease, motor behavior detection, immunohistochemistry and western blot were used to observe and analyze role of PD-1 signaling in brain glial activation, inflammation response and movement dysfunction of Parkinson' animals. It revealed that PD-1/lba1 double-positive microglial cells numerously distributed in control, whereas they were significantly reduced in the MPTP model. Compared with WT-MPTP, KO-MPTP group mice showed significant increases in motor dysfunction, decreased expression level of $\mathrm{TH}$ protein and decrease in $\mathrm{TH}$ positive neuronal protrusions, accompanied by activation of microglial cells and astrocytes, increased expressional levels of proinflammatory cytokine iNOS, TNF- - , IL-1 $\beta$ and IL-6. Further detection showed that PD-1 knockout induced elevated expression and phosphorylation activation of AKT and ERK1/2. The results indicated that PD-1 knockout aggravated animal motor dysfunction of PD model, possibly by 
promoting microglial cell activation, increasing expression of proinflammatory cytokines and triggering AKT and ERK1/2 signaling in the substantia nigra, suggesting that PD-1 signaling abnormality may be involved in micrioglial activation and neuroinflammation response in Parkinson's disease progression.

Many studies showed that abnormal activation of microglial cells and astrocytes in the CNS dominated neuroinflammatory reaction, which might be a critical factor leading to the development of various neurodegenerative diseases such as $A D, M S$ and PD (4-8). In the state of inflammatory reaction, microglial cells functioned as CNS inherent immune cells, activated microglial cells initiated differentiation in two opposite directions, namely, inflammatory state (M1), anti-inflammatory state (M2) functional polarization (29). On the one hand, microglial cells in M2 polarization could remove pathogens or cell fragments and protect from damage to the brains. On the other hand, microglial cell with M1 polarization was in an inflammatory state and could produce a series of cytokines, and thereby further activating astrocytes, being collaborative inflammatory response. The astrocytes regulated the immune response and react to pathological changes by hypertrophy, presented as functional activated state, the activated astrocytes also occurred in similar functional differentiation, that is, inflammatory state (A1) and anti-inflammatory state (A2) polarization, activated A2 astrocytes promoted tissue repair and help maintain function of the central neurons. The activated $A 1$ astrocytes secreted a large number reactive oxygen and pro-inflammatory cytokines, affecting neurons and other glial function, triggering a vicious circle, exacerbating the biological process of amplification of inflammatory reactions and neuronal damage in the CNS $(30,31)$.

It was known that as an important inhibitory immune checkpoint, PD-1 is the regulating molecule of immune cell function (32). Data showed that PD-1 and PD-L1 expression levels changes with healthy and pathological state in the CNS, for example, $20 \%$ of microglial cells expressed PD-L1 in uninfected normal mice, while more than $90 \%$ of microglial cells showed induced PD-L1 expression 1week after infection $(24,25)$. Stimulation of interferon-gama (IFN- $\gamma)$, autoimmune diseases, brain tumors and stroke state induced central T-cell activation and PD-1 production. Increased PD-L1 binding and activation of PD-1 signaling pathway regulated tumor microenvironment and inflammatory response, and thus affected progression of above diseases (33). Some studies indicated that PD1/PD-L1 signaling activation promoted differentiation of microglial cells into anti-inflammatory states (M2) and reduced secondary brain damage in the cerebral hemorrhage $(34,35)$. In addition, many studies focusing on brain tumors showed that tumor cells secreted high level of PD-L1, thereafter induced T cells to produce high level of PD-1 molecule. Combination of PD-1/PD-L1 or PD-1 signaling activation caused decrease or "failure" state of T-cell function, resulting in the migration and diffusion of tumor cells. As a targeted anti-tumor strategy, therefore, PD-1-based inhibitors (PD1 antibody) have attracted more and more attention and successfully used in clinical.

Whether PD-1 signaling pathway was involved in glial cell activation and inflammation of Parkinson's disease, which in turn affected course of the disease, was major concern of this study. Present results showed that the absence state of PD-1 leaded to more obvious glial activation in the MPTP model, increased the expression level of inflammatory factors, and aggravated motor dysfunction, which 
indicated that PD-1 had a certain restrictive effect on neuroinflammation dominated by activated glial cells, and played a neuroprotective role by regulating or limiting inflammatory response. Our result was supported by studies of Yao and other researchers. Yao and other applications used PD-1 knockout and spinal cord injury model, reveal that PD-1 knockout promoted direction of microglial cells and macrophage M1 polarization, and aggravated inflammatory response and neuronal damage (29). Contrary to these observations, Bodhankar used stroke models and found that PD-L1 deficiency improved infarction volume, reduced inflamed cells and inflammatory responses, and improved nerve function (36). It was not clear what causes this difference of study, and it might be related to differences in application of distinct animal models. Other scholars also reported that PD-1 signaling showed a protective role in persistent viral encephalitis. PD-1 signaling activation limited severity of inflammation during acute infection while it maintained a moderate inflammatory response during persistent infection and conduced to resistance to viral re-infection $(37,38)$. It remained a question that if the PD-1 signaling pathway exhibited a "double-edged sword" effect in the different CNS diseases or different state of disease. In addition, it was noticed that PD-1 activation played a protective role by limiting inflammatory response by glial inhibition of T-cell function (39), which was worthy of in-depth study. Further clarification of mechanism of PD-1/PD-L1 signaling pathway in glial cell activation and neuroinflammation shall be helpful to identify new drug intervention targets or establish new Parkinson's disease interventional treatment strategies.

\section{Conclusions}

By using PD-1 knockout mice and MPTP model, this study showed that PD-1 knockout state aggravated animal motor dysfunction of MPTP model by promoting microglial cell activation and neuroinflammatory reaction in the substantia nigra. Data of this study suggested that PD-1 signaling abnormality might be involved in PD pathogenesis or progression.

\section{Abbreviations}

AD, Alzheimer's disease; ARG1, arginase 1; GFAP: glial fibrillary acidic protein; IFNץ: interferon-ү; IL-6, interleukin-6; IL-10, interleukin-10; iNOS, inducible nitric oxide synthase; MPTP, 1-methyl-4-phenyl-1,2,3,6tetrahydropyridine; PD, Parkinson's disease; PD-1, Programmed death receptor-1; PD-L1, Programmed death ligand 1; TGF-b, transforming growth factor-beta; TNF-a: tumor necrosis factor-alpha.

\section{Declarations}

\section{Acknowledgments}

This work was supported by grants from the National Natural Science Foundation of China (31600830, 31371374 and 31760279).

\section{Authors' contributions}


Ying-Ying Cheng, Bei-Yu Chen: Methodology, Writing-original draft preparation.

Gan-Lan Bian: Formal analysis and Validation.

Liang-Wei Chen, Yin-Xiu Ding: Conceptualization, Writing-review \& editing.

\section{Funding}

This research was supported by grants from the National Natural Science Foundation of China (31600830, 31371374 and 31760279). The funding bodies had no role in the design of the study or in the collection, analysis, and interpretation of the data in this manuscript.

\section{Availability of data and materials}

Data and materials from this manuscript are available upon request.

\section{Ethics approval and consent to participate}

Animal experiments were conducted in accordance with the National Institute of Health guide for the care and use of Laboratory animals (NIH Publications, eighth edition revised in 2012) and under the approval of the Animal Experiment Administration Committee of the Fourth Military Medical University.

\section{Consent for publication}

All authors have read the manuscript and approved submission for publication.

\section{Competing interests}

The authors declare that they have no competing interests

\section{References}

1. Armstrong MJ, Okun MS. Diagnosis and treatment of Parkinson disease: A review. JAMA. 2020;323:548-60.

2. Gazerani P. Probiotics for Parkinson's disease. Int J Mol Sci. 2019;20:4121.

3. Poewe W, Seppi K, Tanner CM, Halliday GM, Brundin P, Volkmann J, Schrag AE, Lang AE. Parkinson disease. Nat Rev Dis Primers. 2017;3:17013.

4. Latta CH, Brothers HM, Wilcock DM. Neuroinflammation in Alzheimer's disease, A source of heterogeneity and target for personalized therapy. Neuroscience. 2015;302:103-11.

5. Pajares M, Rojo I, Manda A, Boscá G, Cuadrado L. A. Inflammation in Parkinson's disease: mechanisms and therapeutic implications. Cells. 2020;9(7):1687.

6. Verkhratsky A, Parpura V, Pekna M, Pekny M, Sofroniew M. Glia in the pathogenesis of neurodegenerative diseases. Biochem Soc Trans. 2014;42:1291-301. 
7. Bernaus A, Blanco S, Sevilla A. Glia crosstalk in neuroinflammatory diseases. Front Cell Neurosci. 2020;14:209.

8. Rocha NP, Miranda ASd, Teixeira AL, Insights into neuroinflammation in Parkinson's disease: from biomarkers to anti-inflammatory based therapies. Biomed Res Int 2015; 2015:628192.

9. Masuda T, Sankowski R, Staszewski O, Prinz M. Microglia heterogeneity in the single-cell era. Cell Rep. 2020;30:1271-81.

10. Madore C, Yin ZR, Leibowitz J, Butovsky O. Microglia, lifestyle stress, and neurodegeneration. Immunity. 2020;52:222-40.

11. Khakh BS, Deneen B. The emerging nature of astrocyte diversity. Annu Rev Neurosci. 2019;42:187207.

12. Grigoriadis N, Pesch VV. A basic overview of multiple sclerosis immunopathology. Eur J Neurol. 2015;22(Suppl 2):3-13.

13. Vivekanantham S, Shah S, Dewji R, Dewji A, Khatri C. Rele Ologunde, Neuroinflammation in Parkinson's disease: role in neurodegeneration and tissue repair. Int J Neurosci. 2015;125:717-25.

14. Sochocka M, Diniz BS, Leszek J. Inflammatory Response in the CNS: Friend or Foe? Mol Neurobiol. 2017;54:8071-89.

15. Heneka MT, Kummer MP, Latz E. Innate immune activation in neurodegenerative disease. Nat Rev Immunol. 2014;14:463-77.

16. Ishida $Y$, Agata Y, Shibahara K, Honjo T. Induced expression of PD-1, a novel member of the immunoglobulin gene superfamily, upon programmed cell death. EMBO J. 1992;11:3887-95.

17. Sun C, Mezzadra R, Schumacher TN. Regulation and function of the PD-L1 checkpoint. Immunity. 2018;48:434-52.

18. Balar AV, Weber JS. PD-1 and PD-L1 antibodies in cancer: current status and future directions. Cancer Immunol Immunother. 2017;66:551-64.

19. Ferris R. PD-1 targeting in cancer immunotherapy. Cancer. 2013;119:E1-3.

20. Saresella M, Calabrese E, Marventano I, Piancone F, Gatti A, Farina E, Alberoni M, Clerici M. A potential role for the PD1/PD-L1 pathway in the neuroinflammation of Alzheimer's disease. Neurobiol Aging. 2012;33:624.e11-22.

21. Li PY, Mao LL, Liu XR, Gan Y, Zheng J, Thomson AW, Gao YQ, Chen J, Hu XM. Essential role of program death 1-ligand 1 in regulatory T-cell-afforded protection against blood-brain barrier damage after stroke. Stroke. 2014;45:857-64.

22. Javan MR, Aslani S, Zamani MR, Rostamnejad J, Asadi M, Farhoodi M, Nicknam MH. Downregulation of immunosuppressive molecules, PD-1and PD-L1 but not PD-L2, in the patients with multiple sclerosis. Iran J Allergy Asthma Immunol. 2016;15:296-302.

23. Han RR, Luo JY, Shi YC, Yao Y, Hao JW. PD-L1 (Programmed death ligand 1) protects against experimental intracerebral hemorrhage-induced brain injury. Stroke. 2017;48:2255-62. 
24. Chen Q, Xu LX, Du TJ, Hou YX, Fan WJ, Wu QL, Yan H. Enhanced expression of PD-L1 on microglia after surgical brain injury exerts self-protection from inflammation and promotes neurological repair. Neurochem Res. 2019;44:2470-81.

25. Chauhan P, Lokensgard JR. Glial Cell Expression of PD-L1. Int J Mol Sci, 2019; 20(7).

26. Carter LL, Leach MW, Azoitei ML, Cui JQ, Pelker JW, Jussif J, Benoit S, Ireland G, Luxenberg D, Askew GR, Milarski KL, Groves C, Brown T, Carito BA, Percival K, Carreno BM, Collins M, Marusic S. PD-1/PD$L 1$, but not PD-1/PD-L2, interactions regulate the severity of experimental autoimmune encephalomyelitis. J Neuroimmunol. 2007;182:124-34.

27. Salama AD, Chitnis T, Imitola J, Akiba H, Tushima F, Azuma M, Yagita H, Sayegh MH, Khoury SJ. Critical role of the programmed death-1 (PD-1) pathway in regulation of experimental autoimmune encephalomyelitis. J Exp Med. 2003;198:71-8.

28. Zhu B, Guleria I, Khosroshahi A, Tanuja C, Imitola J, Azuma M, Yagita H, Sayegh MH, Khoury SJ. Differential role of programmed death-ligand 1 and programmed death-ligand 2 in regulating the susceptibility and chronic progression of experimental autoimmune encephalomyelitis. J Immunol. 2006;176:3480-9.

29. Yao AH, Liu FF, Chen K, Tang L, Liu L, Zhang K, Yu CY, Bian GL, Guo HM, Zheng JJ, Cheng P, Ju G, Wang J. Programmed death 1 deficiency induces the polarization of macrophages/microglia to the M1 phenotype after spinal cord injury in mice. Neurotherapeutics. 2014;11:636-50.

30. Rothhammer V, Quintana FJ. Control of autoimmune CNS inflammation by astrocytes. Semin Immunopathol. 2015;37:625-38.

31. Xu JW, Dong HQ, Qian QQ, Zhang X, Wang YW, Jin WJ, Qian YN. Astrocyte-derived CCL2 participates in surgery-induced cognitive dysfunction and neuroinflammation via evoking microglia activation. Behav Brain Res. 2017;332:145-53.

32. Waisman A, Liblau RS, Becher B. Innate and adaptive immune responses in the CNS. Lancet Neurol. 2015;14:945-55.

33. Andrieua GP, Shafrana JS, Smithd CL, Belkina AC, Casey AN, Jafari N, Denis GV. BET protein targeting suppresses the PD-1/PD-L1 pathway in triple-negative breast cancer and elicits anti-tumor immune response. Cancer Lett. 2019;465:45-58.

34. Ansari MJ, Salama AD, Chitnis T, Smith RN, Yagita H, Akiba H, Yamazaki T, Azuma M, Iwai H, Khoury SJ Jr, Sayegh HA. MH, The programmed death-1 (PD-1) pathway regulates autoimmune diabetes in nonobese diabetic (NOD) mice. J Exp Med. 2003;198:63-9.

35. Wu J, Sun L, Li HY, Shen HT, Zhai WW, Yu ZQ, Chen G. Roles of programmed death protein 1 /programmed death-ligand 1 in secondary brain injury after intracerebral hemorrhage in rats: selective modulation of microglia polarization to anti-inflammatory phenotype. $\mathrm{J}$ Neuroinflammation. 2017;14:36.

36. Bodhankar S, Chen YX, Vandenbark AA, Murphy SJ, Offner H. PD-L1 enhances CNS inflammation and infarct volume following experimental stroke in mice in opposition to PD-1. J Neuroinflammation. 2013;10:111. 
37. Ren XF, Akiyoshi K, Vandenbark AA, Hurn PD, Offner H. Programmed death-1 pathway limits central nervous system inflammation and neurologic deficits in murine experimental stroke. Stroke. 2011;42:2578-83.

38. Shwetank, Frost EL, Mockus TE, Ren HM, Toprak M, Lauver MD, Netherby-Winslow CS, Jin G, Cosby $\mathrm{JM}$, Evavold BD, Lukacher AE. PD-1 dynamically regulates inflammation and development of brainresident memory CD8 T Cells During Persistent Viral Encephalitis. Front Immunol. 2019;10:783.

39. Schachtele SJ, Hu SX, Sheng WS, Mutnal MB, Lokensgard GR. Glial cells suppress postencephalitic CD8 + T lymphocytes through PD-L1. Glia. 2014;62:1582-94.

\section{Figures}




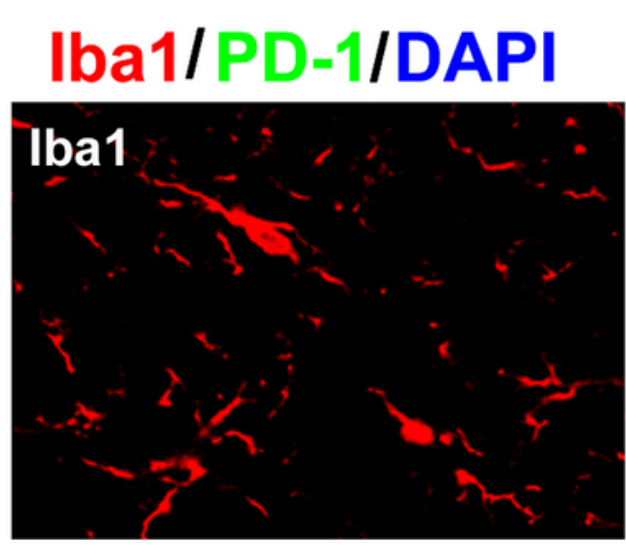

\section{GFAPIPD-1/DAPI}

NeuN/PD-1/DAPI
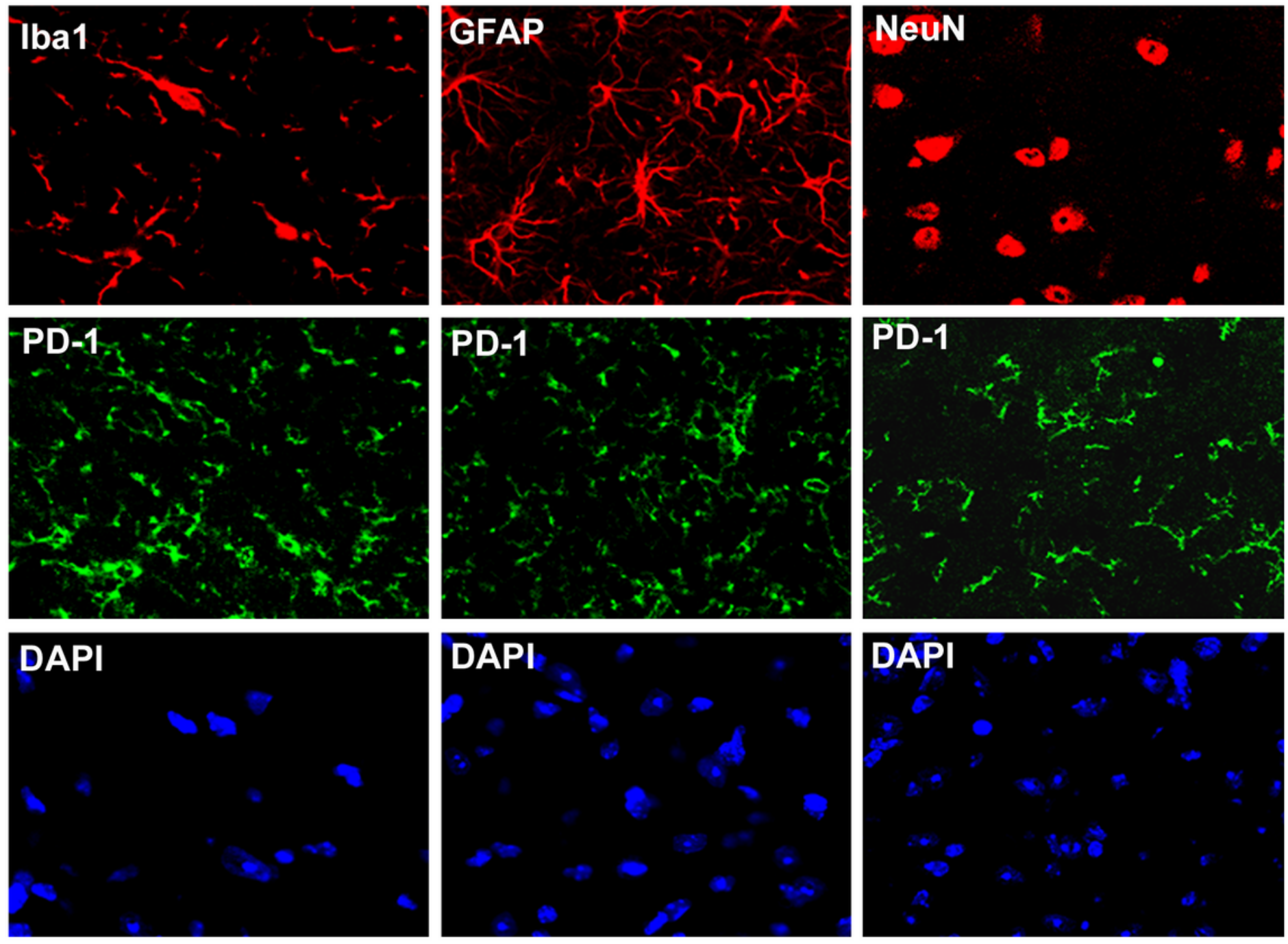

\section{DAPI}

DAPI
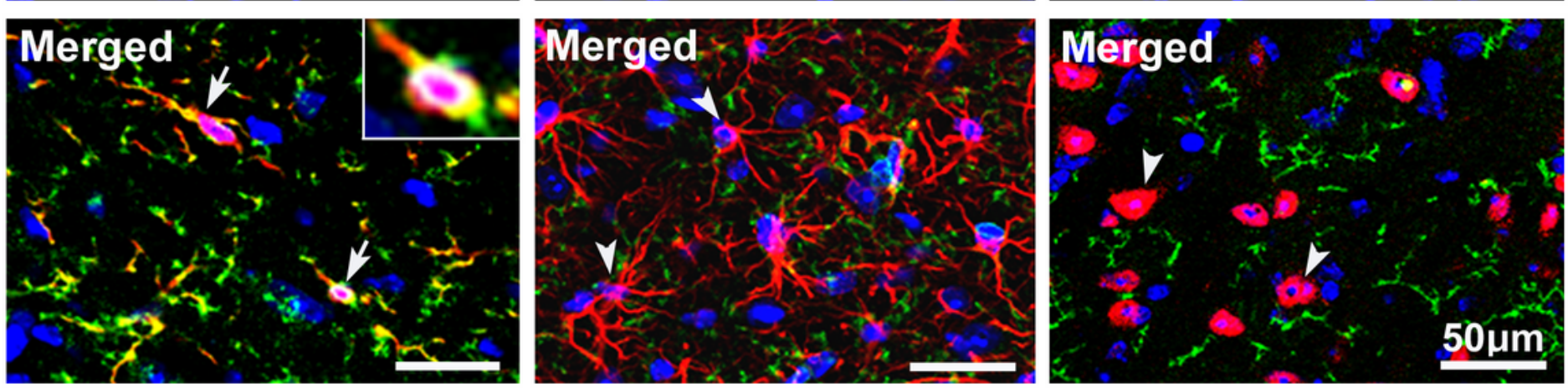

Figure 1

Double immunofluorescence showing PD-1/Iba1, PD-1/GFAP, PD-1/NeuN-positive cells. Distribution of PD-1/lba1 double-positive microglial cells (arrows), GFAP-positive astrocytes (arrowheads), NeuN-positive neurons (arrowheads) are seen in the substantia nigra. 


\section{Control}
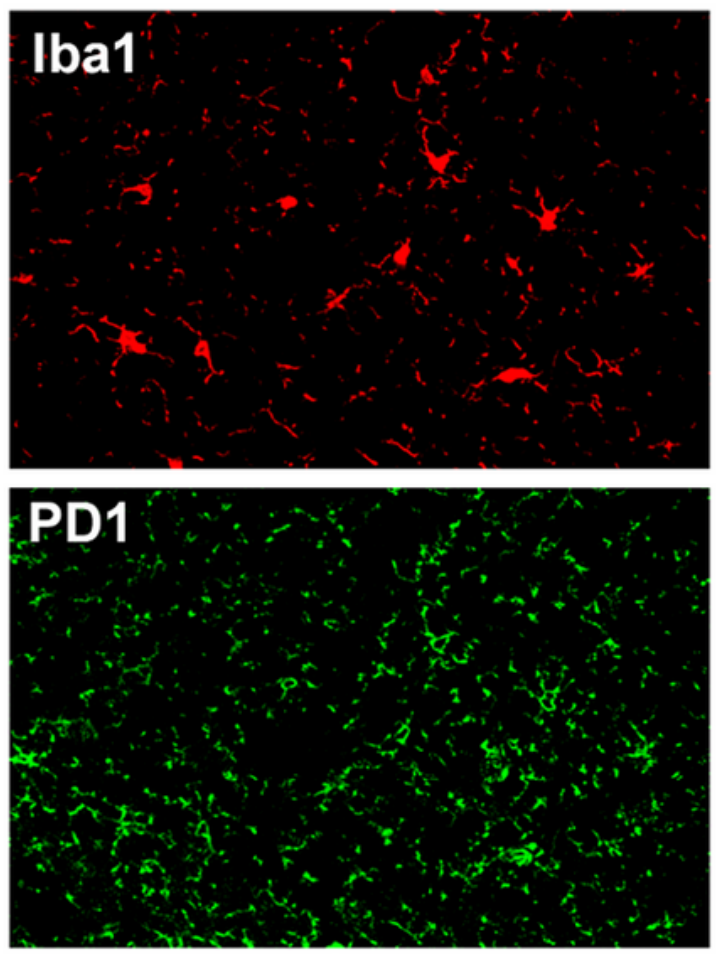

\section{DAPI}
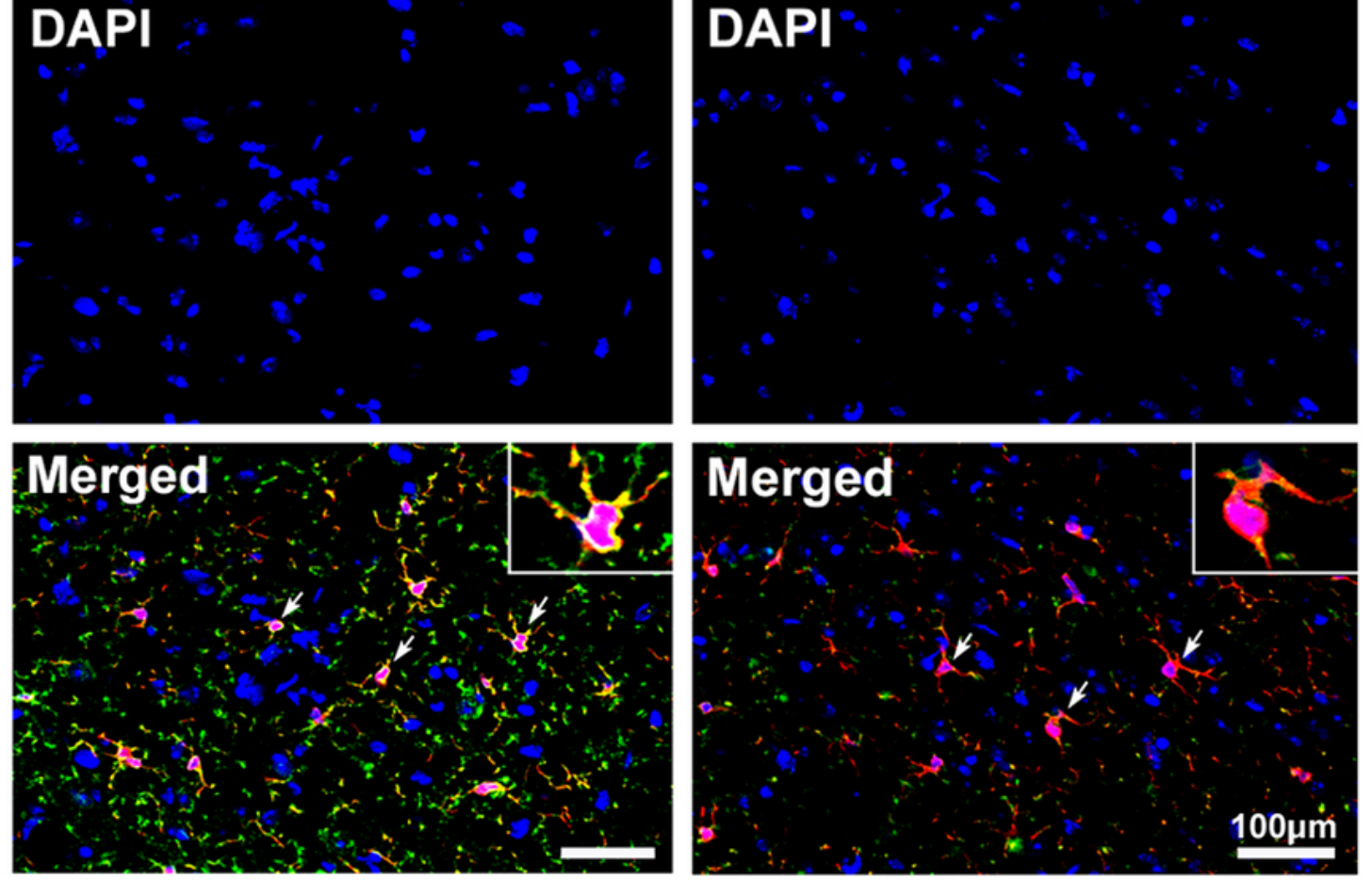

MPTP
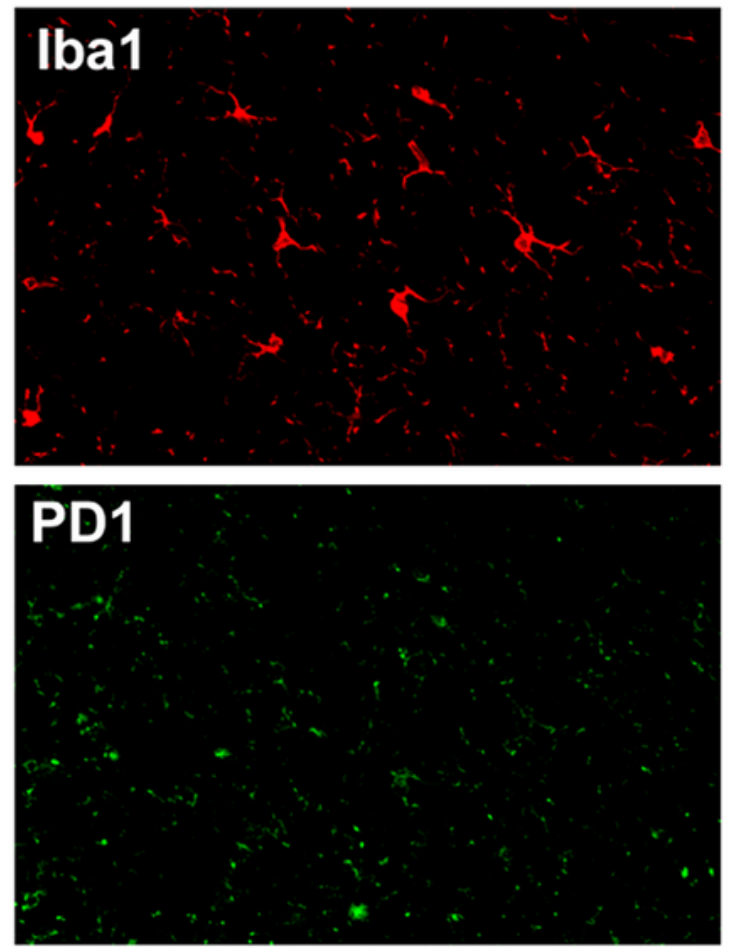

\section{DAPI}

\section{Figure 2}

Morphology and distribution of PD-1/lba1 double-positive microglial cells in the control and MPTP group. PD-1/lba1 double-positive microglial cells are numerously seen in the substantia nigra of control, while they decrease in the MPTP group. PD-1 immunostaining intensity decreases and lba1-positive cell protrusion also becomes shorter and decreased. 
A Day 5
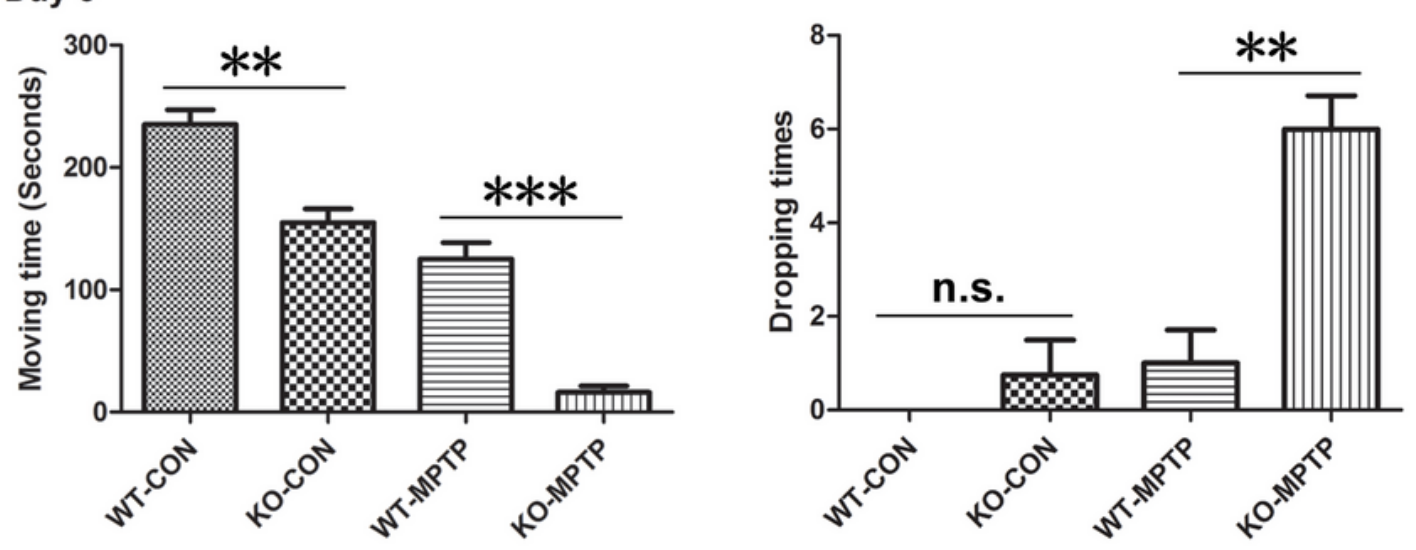

B Day 10
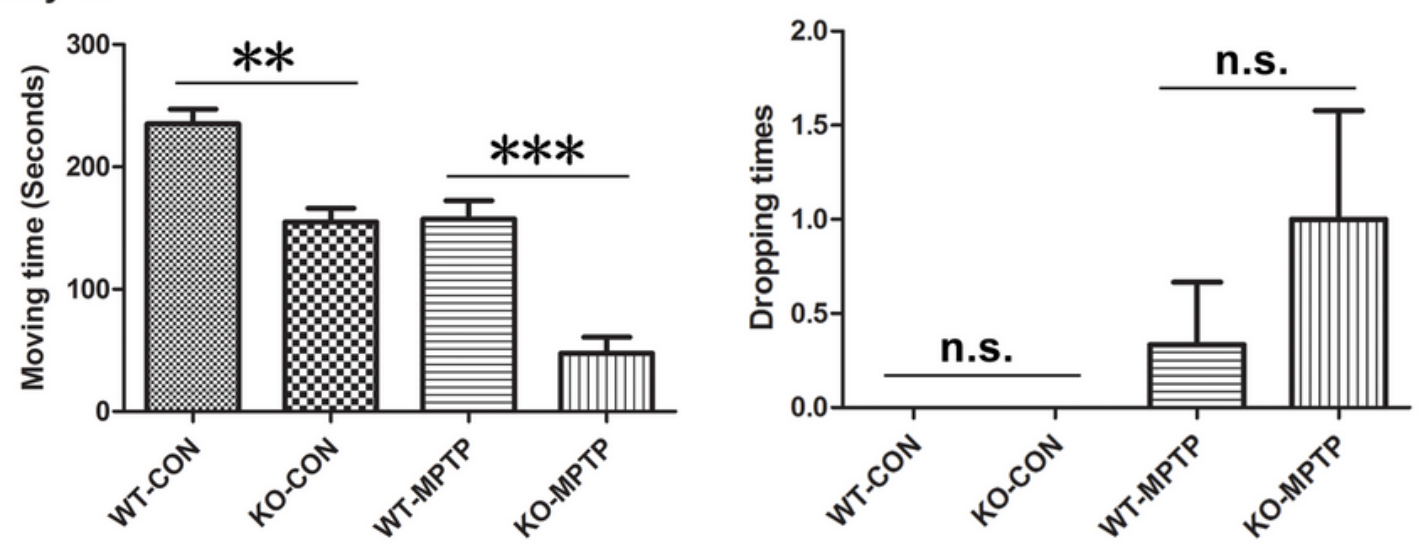

C Day 15
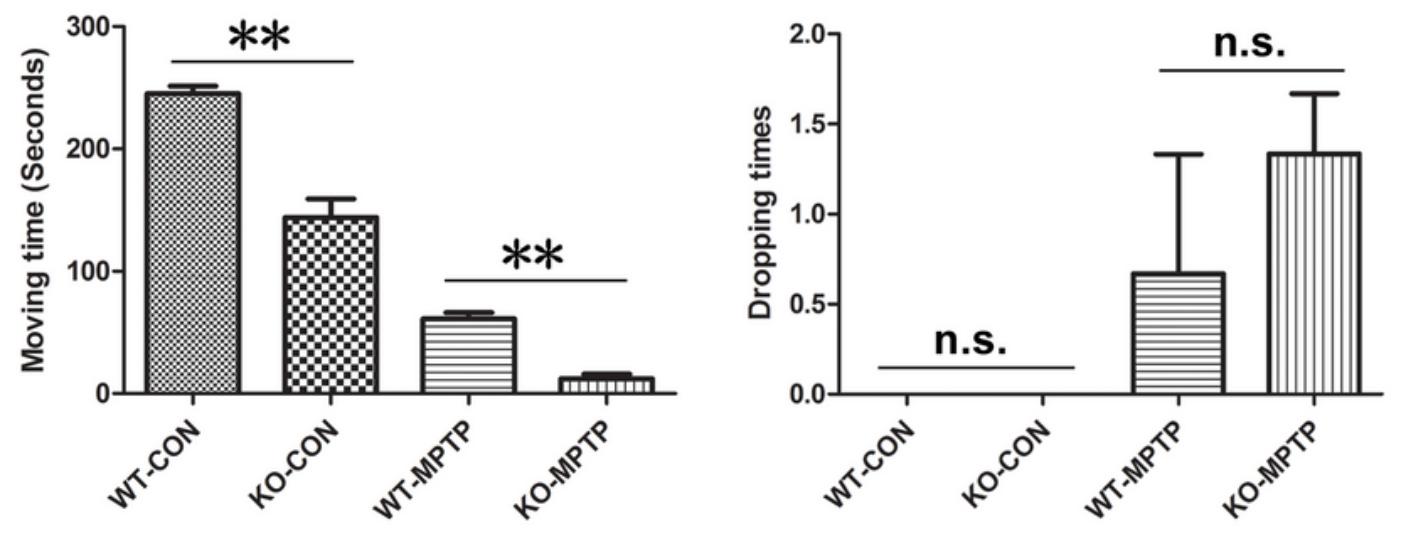

Figure 3

Animal motion behavior detection (climbing rod ability and balance ability) shows movement time (seconds) and fall (times) in WT-CON, KO-CON, WT-MPTP and KO-MPTP group. A-C, Movement time and number of falls in D5, D10 and D15 group, respectively. ANOVA: ${ }^{*} P<0.01, \star \star \star * P<0.001$, n.s. no significance, vs WT-CON or WT-MPTP $(n=5-10)$. 
A

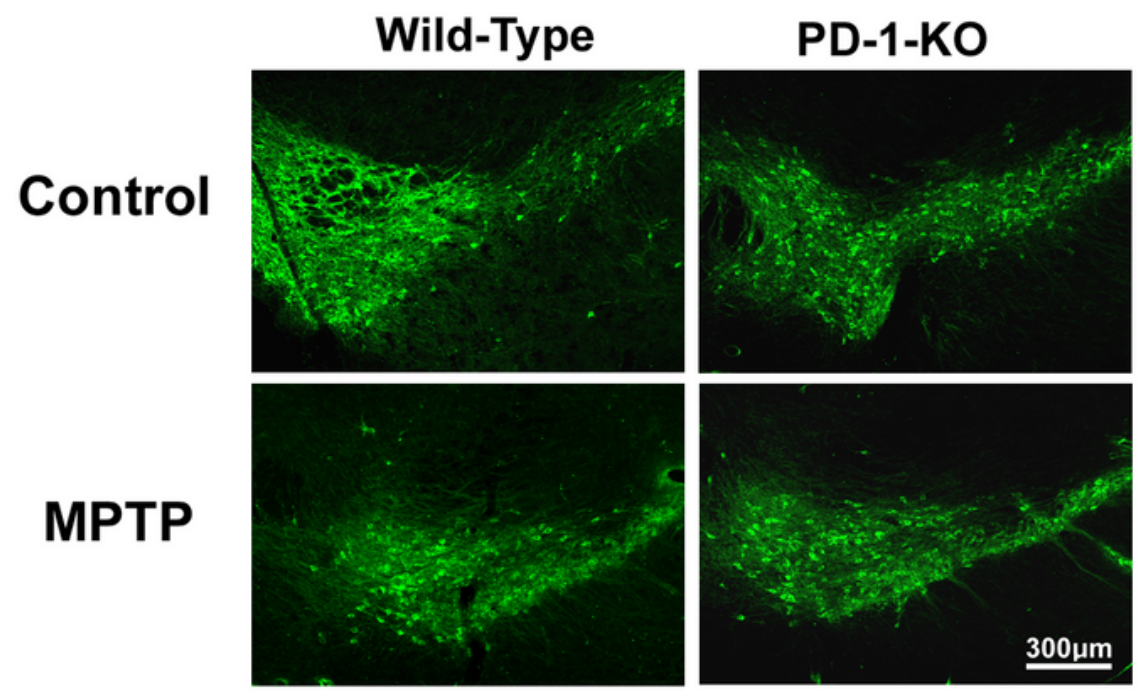

B
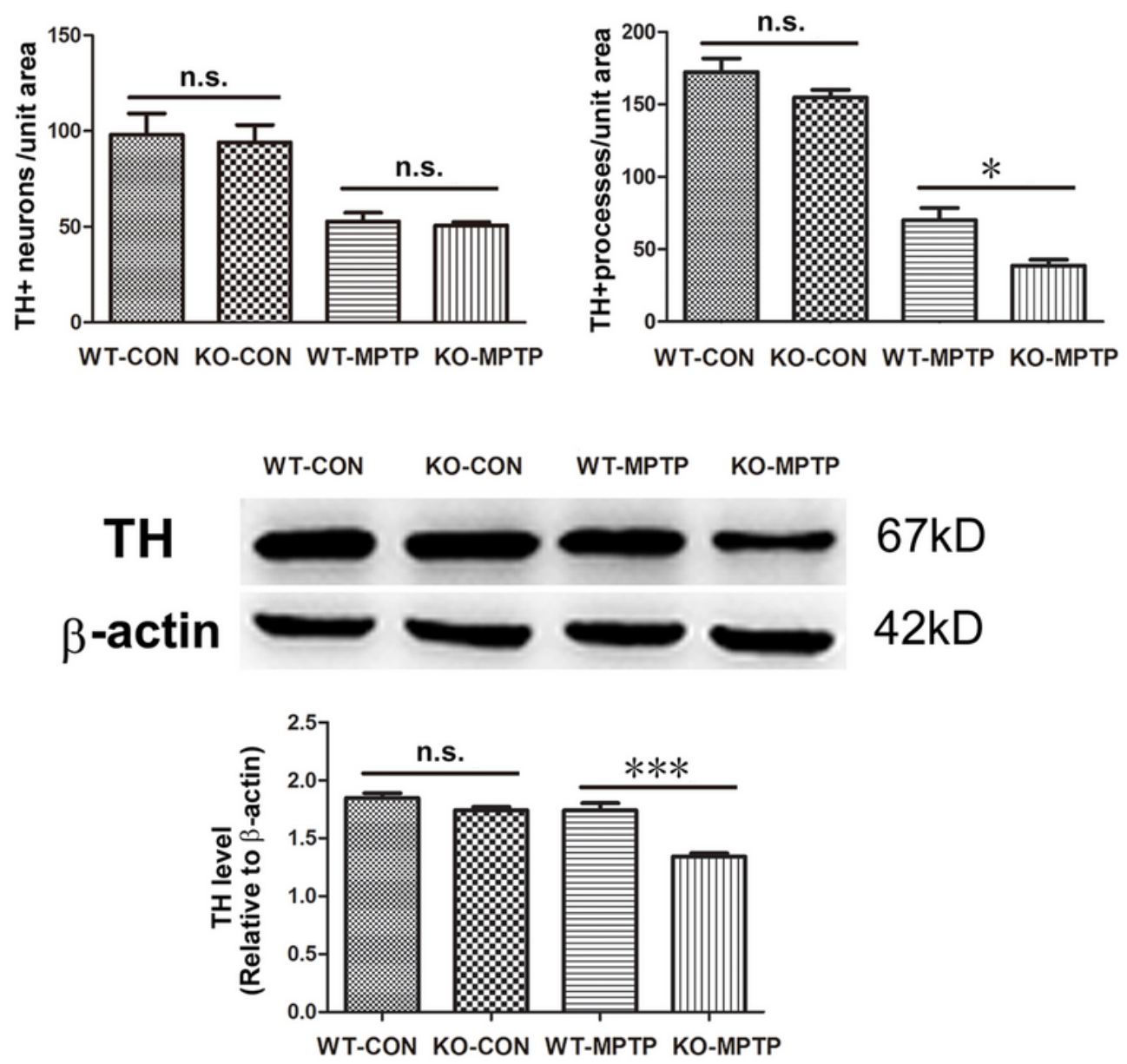

Figure 4

Distribution, quantity of TH-positive dopamine neurons and TH protein expression in WT-CON, KO-CON, WT-MPTP and KO-MPTP group. A, TH-positive dopaminergic neurons in the substantia nigra; $\mathrm{B}$, Comparison of TH-positive neuronal bodies and protrusion; $\mathrm{C}$, Immunoblots of TH expression and comparison of levels among above groups. ANOVA: ${ }^{*} P<0.05,{ }^{*} * P<0.001$, n.s. no significance, vs WT-CON or WT-MPTP $(n=5)$. 
A

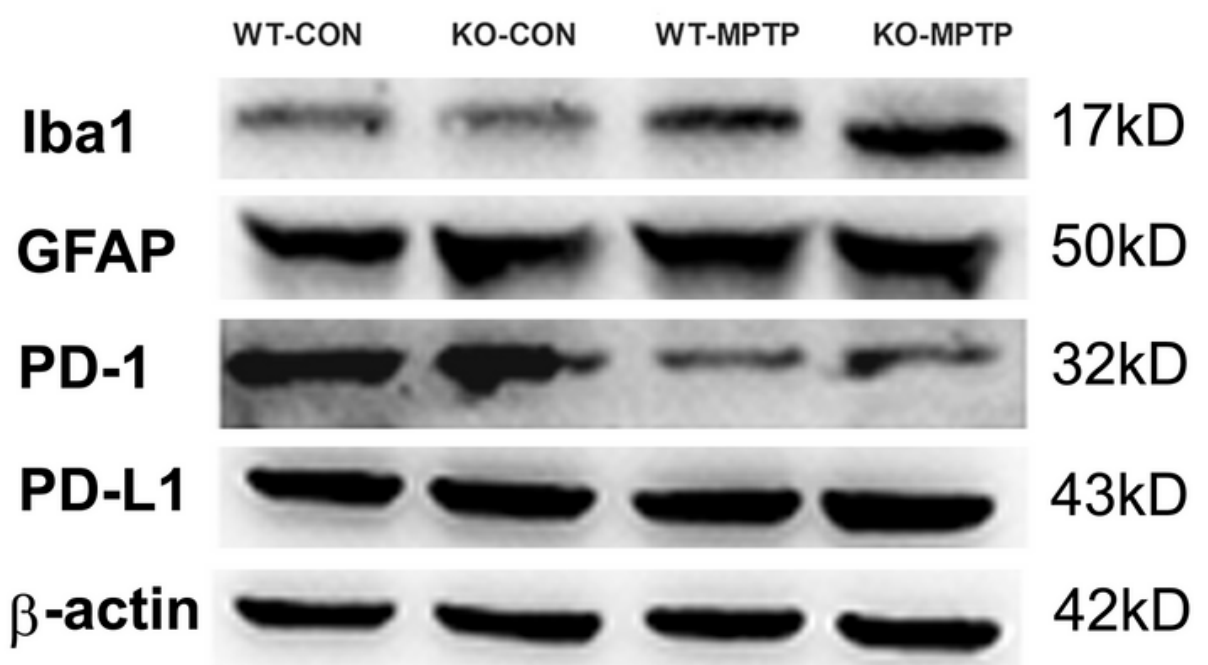

B
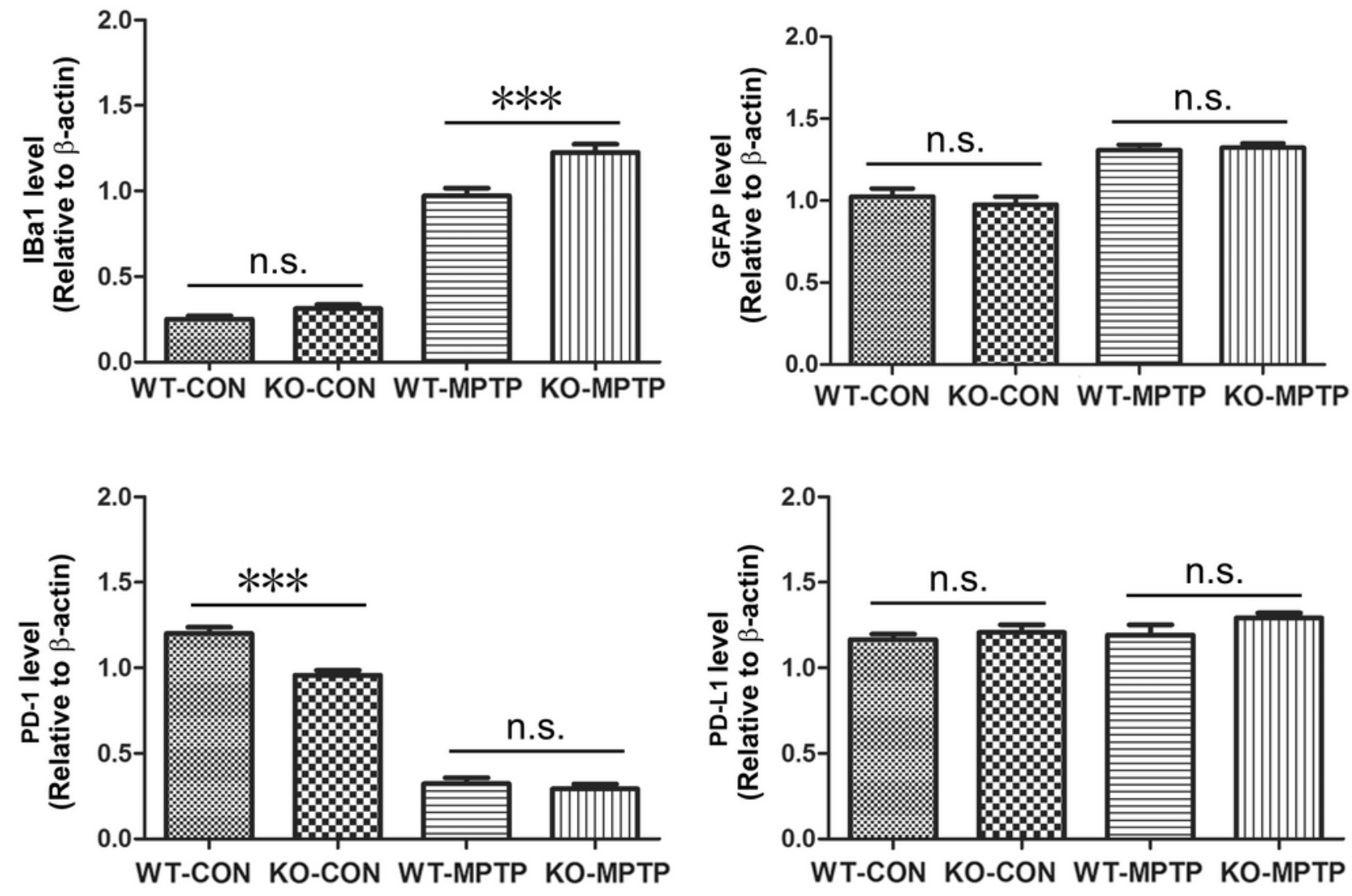

Figure 5

Expression and changes of iba1, GFAP, PD1 and PD-L1 in WT-CON, KO-CON, WT-MPTP and KO-MPTP group. A, Immunoblots of iba1, GFAP, PD1 and PD-L1; B. Comparison of iba1, GFAP, PD1 and PD-L1 level between above four groups. ANOVA: ${ }^{\star \star *} P<0.001$, n.s. no significance, vs WT-CON or WT-MPTP $(n=5)$. 
A
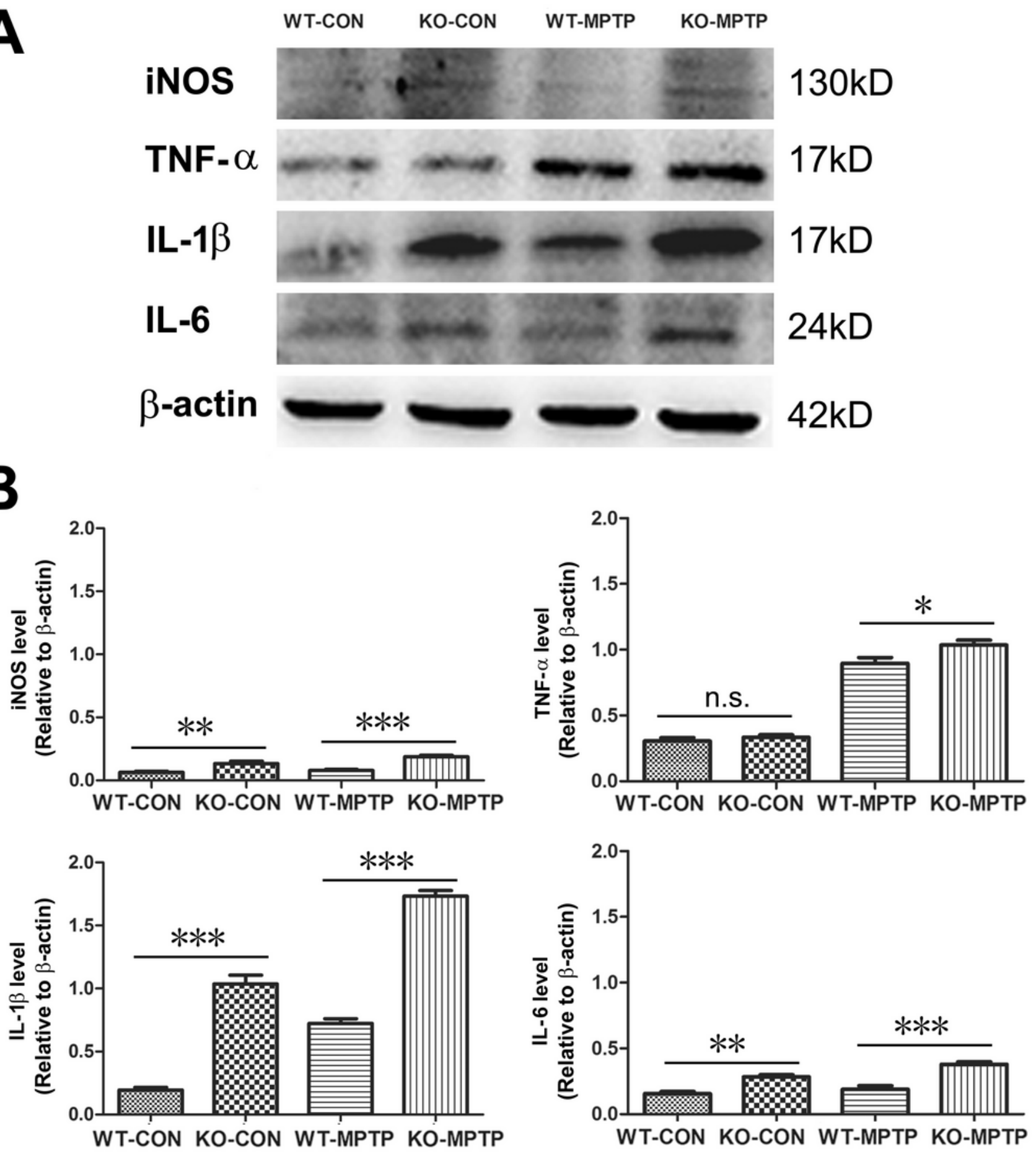

Figure 6

Expression of pro-inflammatory cytokine iNOS, TNF-a, IL-1 $\beta$ and IL6 in WT-CON, KO-CON, WT-MPTP and KO-MPTP group. A, Immunoblots of iNOS, TNF-a, IL-1 $\beta$ and IL6; B. Comparison of iNOS, TNF- $a$, IL-1 $\beta$ and IL6 level among above groups. ANOVA: ${ }^{*} P<0.05,{ }^{* \star} P<0.01$, ${ }^{\star \star *} P<0.001$, n.s. no significance, vs WT-CON or WT-MPTP $(n=5)$. 
A

WT-CON KO-CON WT-MPTP KO-MPTP

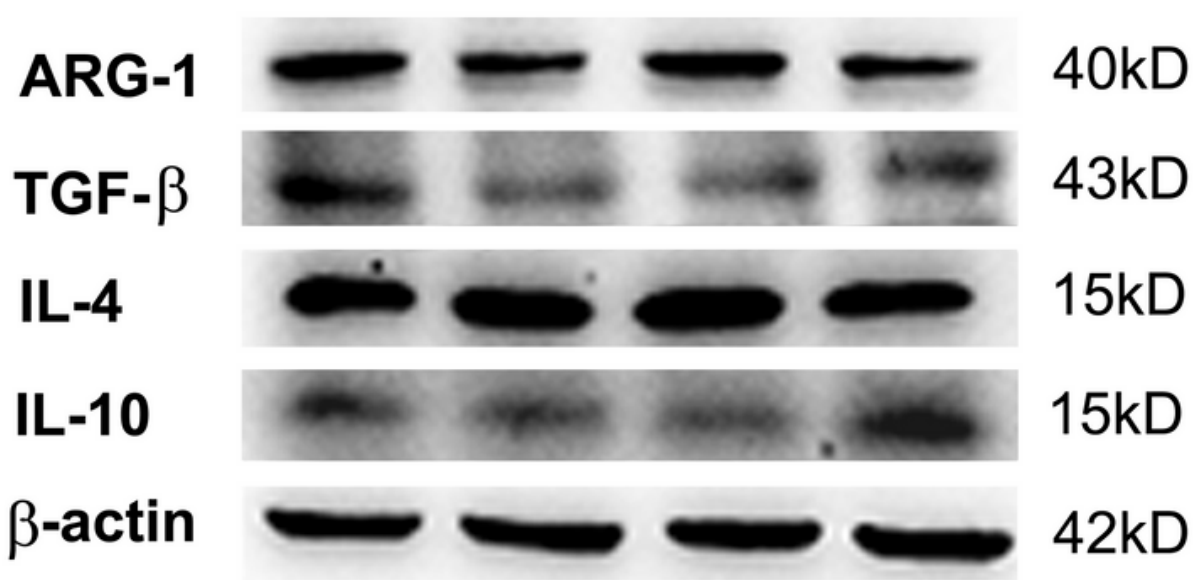

B
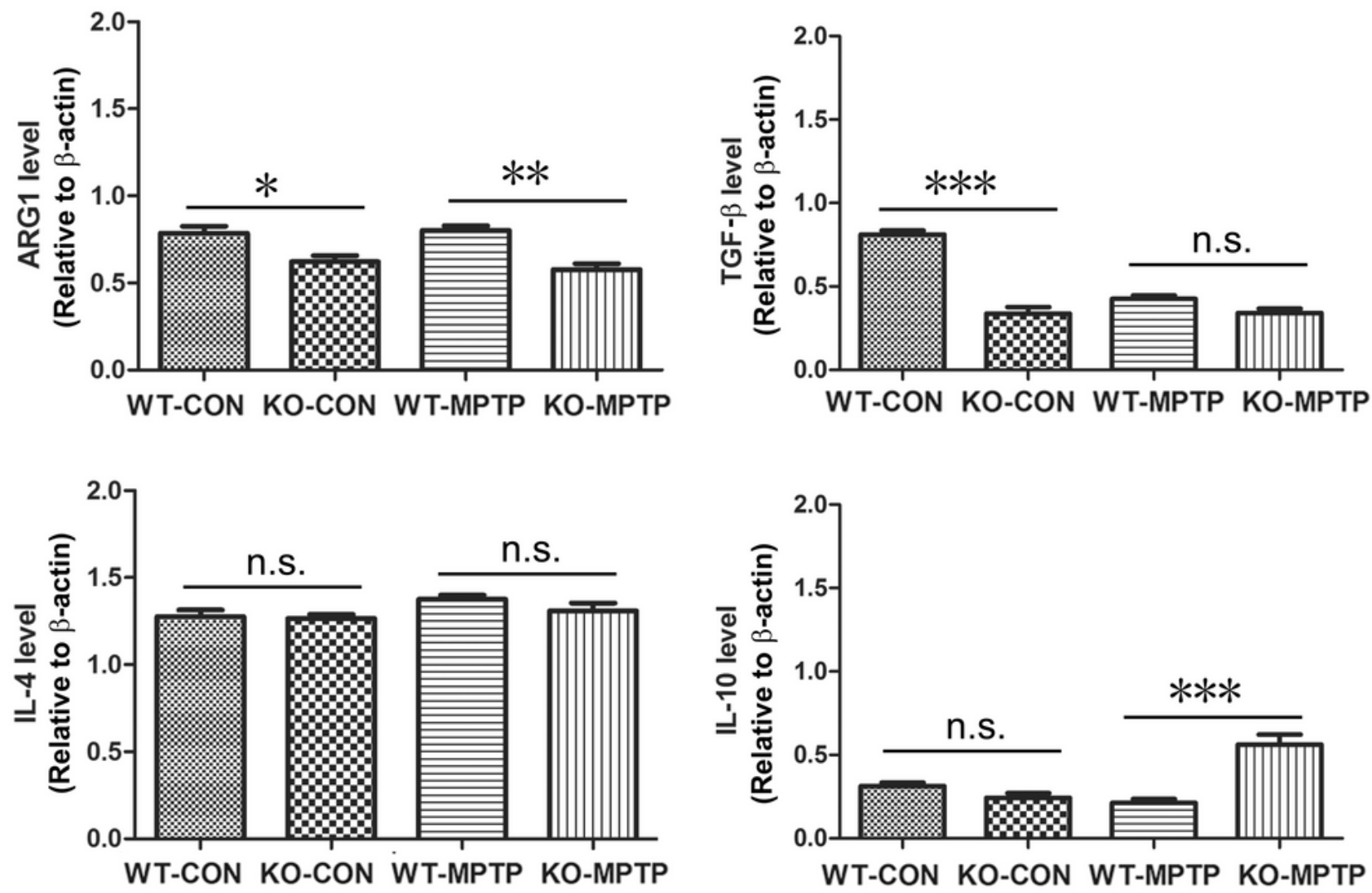

Figure 7

Expression of anti-inflammatory cytokine ARG1, TGF- $\beta$, IL4 and IL10 in WT-CON, KO-CON, WT-MPTP and KO-MPTP group. A, Immunoblots of ARG1, TGF- $\beta$, IL4 and IL10; B. Comparison of ARG1, TGF- $\beta$, IL4 and IL10 level among above groups. ANOV: ${ }^{*} P<0.05,{ }^{\star *} P<0.01$, ${ }^{\star \star \star} P<0.001$, n.s. no significance, vs WT-CON or WT-MPTP $(n=5)$. 
A
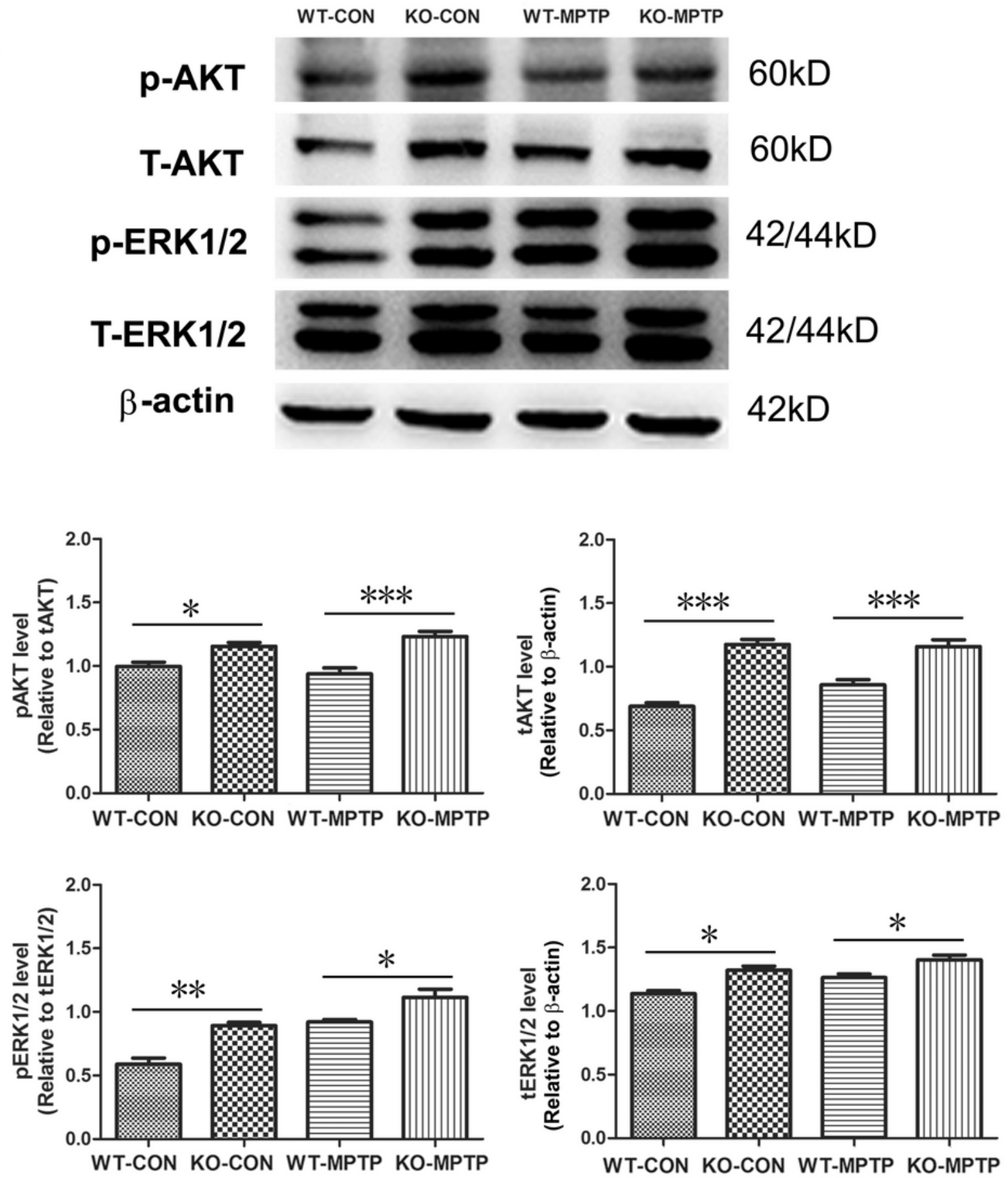

Figure 8

Expression of anti-inflammatory cytokine ARG1, TGF- $\beta$, IL4 and IL10 in WT-CON, KO-CON, WT-MPTP and KO-MPTP group. A, Immunoblots of ARG1, TGF- $\beta$, IL4 and IL10; B. Comparison of ARG1, TGF- $\beta$, IL4 and IL10 level among above groups. ANOV: ${ }^{*} P<0.05,{ }^{\star *} P<0.01$, ${ }^{\star \star \star} P<0.001$, n.s. no significance, vs WT-CON or WT-MPTP $(n=5)$. 